T. Ono

Nagoya Math. J.

Vol. 95 (1984), 63-71

\title{
ON DEFORMATIONS OF HOPF MAPS AND HYPERGEOMETRIC SERIES
}

\author{
TAKASHI ONO
}

\section{Introduction}

Let $\boldsymbol{R}^{n}$ denote the Euclidean space of dimension $n \geqq 1$ with the standard inner product $\langle x, y\rangle$ and the norm $N x=\langle x, x\rangle$. We shall denote by $d \omega_{n-1}$ the volume element of the unit sphere $S^{n-1}=\left\{x \in \boldsymbol{R}^{n} ; N x=1\right\}$ normalized so that the volume of $S^{n-1}$ is 1 .

With each continuous map $f: S^{n-1} \rightarrow \boldsymbol{R}^{m}$, we shall associate a function $f^{*}(z)$ of a complex variable $z$ by

$$
f^{\sharp}(z)=\int_{S^{n-1}} e^{z(f(x))} d \omega_{n-1} .
$$

Clearly $f^{\sharp}(z)$ is an entire function and its Taylor expansion is given by

$$
f^{\sharp}(z)=\sum_{k=0}^{\infty} N_{k}(f) \frac{z^{k}}{k !}
$$

where

$$
N_{k}(f)=\int_{S^{n-1}} N(f(x))^{k} d \omega_{n-1} .
$$

When $f$ is spherical, i.e. when $f$ maps $S^{n-1}$ in $S^{m-1}$, we have $f^{\sharp}(z)=e^{z}$. When we are given a family $\left\{f_{t}\right\}, 0 \leqq t \leqq 1$, of maps: $S^{n-1} \rightarrow \boldsymbol{R}^{m}$ such that $f_{0}$ is spherical, we have a family $\left\{f_{t}^{\sharp}\right\}$ of entire functions beginning with $f_{0}^{\sharp}=e^{z}$ and ending with some advanced function $f_{1}^{*}$.

Here is an illustrative example: consider the family

$$
f_{t}(x)=\left(x_{1}^{2}-x_{2}^{2}, 2(1+t)^{1 / 2} x_{1} x_{2}\right), \quad 0 \leqq t \leqq 1 .
$$

The map $f_{0}: S^{1} \rightarrow \boldsymbol{R}^{2}$ is spherical since it is the squaring $x \mapsto x^{2}$ in $C=\boldsymbol{R}^{2}$. Passing to the polar coordinates, we have $N\left(f_{t}(x)\right)=1+\mathrm{t} \sin ^{2} 2 \theta$ and

Received March 2, 1983. 


$$
\begin{aligned}
f_{t}^{\sharp}(z) e^{-z} & =\frac{1}{2 \pi} \int_{0}^{2 \pi} e^{z t \sin ^{2} 2 \theta} d \theta=\sum_{k=0}^{\infty} \frac{1 \cdot 3 \cdot 5 \cdots(2 k-1)}{2 \cdot 4 \cdot 6 \cdots 2 k} \frac{(t z)^{k}}{k !} \\
& ={ }_{1} F_{1}(1 / 2 ; 1 ; t z)
\end{aligned}
$$

a Kummer's hypergeometric series.

The purpose of this paper is to find similar relations between certain deformations of classical Hopf fibrations $S^{3} \rightarrow S^{2}, S^{7} \rightarrow S^{4}, S^{15} \rightarrow S^{8}$ and Kummer's hypergeometric series.

\section{$\S 1$. Prerequisites}

As for proofs of formulas below, see our earlier paper [2].

The symbols $Z, \boldsymbol{Q}, \boldsymbol{R}, \boldsymbol{C}, \boldsymbol{H}, \boldsymbol{O}$ denote the set of integers, rational numbers, real numbers, complex numbers, Hamilton's quaternions and Cayley's octonions, respectively. The set of nonnegative real numbers is denoted by $\boldsymbol{R}_{+}$. For a subset $M$ of $\boldsymbol{R}$, we put $M_{+}=M \cap \boldsymbol{R}_{+}$. The set of all $(m \times n)$-matrices over a field $K$ is written $K_{m, n}$. If $m=n$, we write $K_{m}$ for $K_{m, m}$. For a symmetric matrix $A \in K_{n}$ and vectors $x \in K^{n}$, we put $A[x]={ }^{t} x A x$, the quadratic form of $A$. For $a \in C, k \in Z_{+}$, the Appell's symbol is:

$$
(a, k)=\left\{\begin{array}{cl}
a(a+1) \cdots(a+k-1) & \text { if } k \geqq 1 \\
1 & \text { if } k=0 .
\end{array}\right.
$$

We have the duplication formula: $(2 a, 2 k)=4^{k}(a, k)(a+1 / 2, k)$. For $a=$ $\left(a_{1}, \cdots, a_{p}\right) \in C^{p}, \quad b=\left(b_{1}, \cdots, b_{q}\right) \in C^{q}$, the (generalized) hypergeometric series is defined by

$$
{ }_{p} F_{q}(a ; b ; z)=\sum_{k=0}^{\infty} \frac{\left(a_{1}, k\right) \cdots\left(a_{p}, k\right)}{\left(b_{1}, k\right) \cdots\left(b_{q}, k\right)} \frac{z^{k}}{k !} .
$$

${ }_{2} F_{1}$ and ${ }_{1} F_{1}$ are also called Gauss' and Kummer's series, respectively. For $\lambda=\left(\lambda_{1}, \cdots, \lambda_{n}\right) \in \boldsymbol{R}^{n}$ and $\nu \in Z_{+}$, the numbers $b_{\nu}(2 ; \lambda)$ are defined by the generating relation:

$$
\sum_{\nu=0}^{\infty} b_{\nu}(2 ; \lambda) t^{\nu}=\prod_{i=1}^{n}\left(1-4 \lambda_{i} t\right)^{-1 / 2}
$$

In particular, we have

$$
b_{\nu}\left(2 ; 1_{n}\right)=\frac{4^{\nu}(n / 2, \nu)}{\nu !} \quad \text { for } 1_{n}=(1, \cdots, 1) \in Z_{+}^{n} .
$$


When $\lambda=\left(\lambda_{1}, \cdots, \lambda_{n}\right)$ is the set of eigenvalues of a quadratic form $q(x)$ on $\boldsymbol{R}^{n}$, we have

$$
\int_{S^{n-1}} q(x)^{\nu} d \omega_{n-1}=\frac{b_{\nu}(2 ; \lambda)}{b_{\nu}\left(2 ; 1_{n}\right)} .
$$

For a continuous map $f: S^{n-1} \rightarrow \boldsymbol{R}^{m}$, we put

$$
\begin{aligned}
& f_{\nu}(\xi)=\int_{S^{n-1}}\langle\xi, f(x)\rangle^{\nu} d \omega_{n-1}, \quad \xi \in \boldsymbol{R}^{m}, \\
& \sigma_{\nu}(f)=\int_{S^{m-1}} f_{\nu}(\xi) d \omega_{m-1}, \quad \nu \in Z_{+} .
\end{aligned}
$$

Then, we have

$$
N_{k}(f)=\int_{S^{n-1}} N(f(x))^{k} d \omega_{n-1}=\frac{b_{k}\left(2 ; 1_{m}\right)}{b_{k}(2 ; 1)} \sigma_{2 k}(f) \quad k \in Z_{+} .
$$

\section{§2. Quadratic maps of type (S)}

Let $f: \boldsymbol{R}^{n} \rightarrow \boldsymbol{R}^{m}$ be a quadratic map. By definition, each component $f_{i}(x), 1 \leqq i \leqq m$, of $f(x)$ is a quadratic form on $\boldsymbol{R}^{n}$ and we can write $f_{i}(x)$ $=A_{i}[x]$ with a symmetric matrix $A_{i}$ in $\boldsymbol{R}_{n}$. We shall obtain a general formula for the number $N_{k}(f)$. In view of (1.4), (1.5), (1.6), we shall consider $f_{2 k}(\xi)$ and $\sigma_{2 k}(f)$ in order. Since $\langle\xi, f(x)\rangle=\xi_{1} f_{1}(x)+\cdots+\xi_{m} f_{m}(x)$ $=\xi_{1} A_{1}[x]+\cdots+\xi_{m} A_{m}[x]$, we have

$$
\langle\xi, f(x)\rangle=A[x] \quad \text { with } A=\xi_{1} A_{1}+\cdots+\xi_{m} A_{m} .
$$

Let $\lambda=\left(\lambda_{1}, \cdots, \lambda_{n}\right)$ be the eigenvalues of $A$. Then, by (1.3), (1.4) we have

$$
f_{2 k}(\xi)=\frac{b_{2 k}(2 ; \lambda)}{b_{2 k}\left(2 ; 1_{n}\right)} .
$$

From (1.3), (1.5), (1.6), (2,2), it follows that

$$
N_{k}(f)=\beta_{k} \int_{S^{m-1}} b_{2 k}(2 ; \lambda) d \omega_{m-1}
$$

where

$$
\beta_{k}=\frac{b_{k}\left(2 ; 1_{m}\right)}{b_{k}(2 ; 1) b_{2 k}\left(2 ; 1_{n}\right)} .
$$

Using (1.2) three times and the duplication formula for Appell's symbol twice, we can determine $\beta_{k}$ explicitly and we get 


$$
N_{k}(f)=\frac{(m / 2, k) k !}{4^{2 k}(n / 4, k)((n+2) / 4, k)} \int_{S^{m-1}} b_{2 k}(2 ; \lambda) d \omega_{m-1} .
$$

In view of (1.1), the main problem is to determine the eigenvalues of the symmetric matrix $A=\xi_{1} A_{1}+\cdots+\xi_{m} A_{m}$. In order to facilitate the argument, let us make the following assumptions on the quadratic map $f: \boldsymbol{R}^{n} \rightarrow \boldsymbol{R}^{m}$ in terms of the matrix $A$ in $(2,1)$.

(2.4) Definition. We shall say that a quadratic map $f: \boldsymbol{R}^{n} \rightarrow \boldsymbol{R}^{m}$ is of type (S) if the following conditions are satisfied:

(S1) $n$ is even: $n=2 p$,

(S2) the trace of $A$ is zero,

(S3) $A^{2}=a 1_{2 p}$ where $a=a(\xi)$ is a positive definite quadratic form on $\boldsymbol{R}^{m}$.

(2.5) Proposition. Suppose that $f: \boldsymbol{R}^{2 p} \rightarrow \boldsymbol{R}^{m}$ is a quadratic map of type (S). Notation being as in (2.4), let $\lambda=\left(\lambda_{1}, \cdots, \lambda_{2 p}\right)$ be the set of eigenvalues of $A$. Then, after a necessary arrangement of $\lambda_{i}$ 's, we have $\lambda_{1}=$ $\cdots=\lambda_{p}=\sqrt{a}$ and $\lambda_{p+1}=\cdots=\lambda_{2 p}=-\sqrt{a}$.

Proof. Let $T$ be an orthogonal matrix in $\boldsymbol{R}_{2 p}$ such that

$$
{ }^{t} T A T=\left(\begin{array}{lll}
\lambda_{1} & & \\
& \ddots & \\
& & \lambda_{2 p}
\end{array}\right) .
$$

By (S3) we have $\left({ }^{t} T A T\right)^{2}={ }^{t} T A^{2} T=a 1_{2 p}$ and so $\lambda_{i}^{2}=a$ for all $i, 1 \leqq i \leqq 2 p$. Our assertion then follows at once from (S2).

Q.E.D.

Now, back to the formula (2.3), if $f$ is of type (S), we have, by (1.1),

$$
\begin{aligned}
\sum_{\nu=0}^{\infty} b_{\nu}(2 ; \lambda) t^{\nu} & =(1-4 \sqrt{a t})^{-p / 2}(1+4 \sqrt{a} t)^{-p / 2} \\
& =\left(1-4^{2} a t^{2}\right)^{-p / 2}=\sum_{k=0}^{\infty}\left(\frac{p}{2}, k\right) \frac{4^{2 k} a^{k}}{k !} t^{2 k}
\end{aligned}
$$

and so

$$
b_{2 k}(2 ; \lambda)=\left(\frac{p}{2}, k\right) \frac{4^{2 k} a^{k}}{k !}, \quad k \in Z_{+}
$$

Combining (2.3) and (2.6), with $n=2 p$, we get

$$
N_{k}(f)=\frac{(m / 2, k)}{((p+1) / 2, k)} \int_{S^{m-1}} a^{k} d \omega_{m-1} \quad \text { when } f \text { is of type (S) . }
$$


Call $\mu=\left(\mu_{1}, \cdots, \mu_{m}\right)$ the eigenvalues of $a=a(\xi)$; by (S3) all $\mu_{i}$ are positive. From (1.2), (1.3), (2.7), it follows that

$$
N_{k}(f)=\frac{k !}{4^{k}((p+1) / 2, k)} b_{k}(2 ; \mu) \quad \text { when } f \text { is of type (S). }
$$

A further determination of $N_{k}(f)$ depends on $\mu$, the eigenvalues of $a=a(\xi)$, via (1.1), again.

As an illustrative example, let us consider the case where $p=1$, $m=2$, i.e. the case of a pair of binary quadratic forms:

$$
f(x)=\left(\begin{array}{l}
f_{1}(x) \\
f_{2}(x)
\end{array}\right)=\left(\begin{array}{l}
\alpha_{1} x_{1}^{2}+2 \beta_{1} x_{1} x_{2}-\alpha_{1} x_{2}^{2} \\
\alpha_{2} x_{1}^{2}+2 \beta_{2} x_{1} x_{2}-\alpha_{2} x_{2}^{2}
\end{array}\right) .
$$

Thus, we have

$$
A_{1}=\left(\begin{array}{rr}
\alpha_{1} & \beta_{1} \\
\beta_{1} & -\alpha_{1}
\end{array}\right), \quad A_{2}=\left(\begin{array}{rr}
\alpha_{2} & \beta_{2} \\
\beta_{2} & -\alpha_{2}
\end{array}\right)
$$

and $A=\xi_{1} A_{1}+\xi_{2} A_{2}$. Clearly the trace of $A$ is zero. If we assume that $\alpha_{1} \beta_{2}-\alpha_{2} \beta_{1} \neq 0$, one verifies easily that $f$ is of type (S) with

$$
A^{2}=a(\xi)=\left(\alpha_{1}^{2}+\beta_{1}^{2}\right) \xi_{1}^{2}+2\left(\alpha_{1} \alpha_{2}+\beta_{1} \beta_{2}\right) \xi_{1} \xi_{2}+\left(\alpha_{2}^{2}+\beta_{2}^{2}\right) \xi_{2}^{2},
$$

this being positive definite. The characteristic polynomial of the matrix of $a(\xi)$ is

$$
t^{2}-\left(\alpha_{1}^{2}+\alpha_{2}^{2}+\beta_{1}^{2}+\beta_{2}^{2}\right) t+\left(\alpha_{1} \beta_{2}-\alpha_{2} \beta_{1}\right)^{2} .
$$

Now, by (1.1), we have

$$
\begin{aligned}
\sum_{k=0}^{\infty} b_{k}(2 ; \mu) t^{k} & =\left(1-4 \mu_{1} t\right)^{-1 / 2}\left(1-4 \mu_{2} t\right)^{-1 / 2} \\
& =\left(1-4\left(\mu_{1}+\mu_{2}\right) t+\mu_{1} \mu_{2}(4 t)^{2}\right)^{-1 / 2}=\left(1-2 x z+z^{2}\right)^{-1 / 2}
\end{aligned}
$$

with $x=\left(\mu_{1}+\mu_{2}\right) / 2\left(\mu_{1} \mu_{2}\right)^{1 / 2}, \quad z=4\left(\mu_{1} \mu_{2}\right)^{1 / 2} t$. In view of the well-known generating relation of the Legendre polynomials;

$$
\left(1-2 x z+z^{2}\right)^{-1 / 2}=\sum_{k=0}^{\infty} P_{k}(x) z^{k},
$$

we have

$$
b_{k}(2 ; \mu)=4^{k}\left(\mu_{1} \mu_{2}\right)^{k / 2} P_{k}\left(\frac{\mu_{1}+\mu_{2}}{2\left(\mu_{1} \mu_{2}\right)^{1 / 2}}\right) .
$$

From (2.8), (2.10), (2.11), it follows that 


$$
N_{k}(f)=\left|\alpha_{1} \beta_{2}-\alpha_{2} \beta_{1}\right|^{k} P_{k}\left(\frac{\alpha_{1}^{2}+\alpha_{2}^{2}+\beta_{1}^{2}+\beta_{2}^{2}}{2\left|\alpha_{1} \beta_{2}-\alpha_{2} \beta_{1}\right|}\right)
$$

For simplicity, put $\Delta=\left|\alpha_{1} \beta_{2}-\alpha_{2} \beta_{1}\right|$ and $\sigma=\alpha_{1}^{2}+\alpha_{2}^{2}+\beta_{1}^{2}+\beta_{2}^{2}$. Then, we have

$$
\begin{aligned}
f^{\#}(z) & =\sum_{k=0}^{\infty} N_{k}(f) \frac{z^{k}}{k !}=\sum_{k=0}^{\infty} P_{k}\left(\frac{\sigma}{2 \Delta}\right) \frac{(\Delta z)^{k}}{k !} \\
& =e^{\sigma z / 2}{ }_{0} F_{1}\left(; 1 ;\left(\frac{z}{4}\right)^{2}\left(\sigma^{2}-4 \Delta^{2}\right)\right) \cdot{ }^{1)}
\end{aligned}
$$

If, in particular, $\alpha_{1}=1, \alpha_{2}=0, \beta_{1}=0, \beta_{2}=(1+t)^{1 / 2}$, then $\sigma=2+t, \Delta=$ $(1+t)^{1 / 2}$ and we get

$$
f^{\sharp}(z)=f_{t}^{\sharp}(z)=e_{0}^{(1+t / 2) z} F_{1}\left(; 1 ;\left(\frac{t z}{4}\right)^{2}\right)
$$

which is consistent with the formula $f_{t}^{\sharp}(z)=e^{z}{ }_{1} F_{1}(1 / 2 ; 1 ; t z)$ of the example in the introduction as can be verified directly.

\section{§3. Deformations of Hopf maps}

Throughout this section, we shall denote by $X$ one of the algebras $\boldsymbol{R}, \boldsymbol{C}, \boldsymbol{H}, \boldsymbol{O}$, of real numbers, complex numbers, Hamilton's quaternions and Cayley's octonions, respectively. Using the standard basis, $X$ may be identified with the Euclidean space, $\boldsymbol{R}^{p}, p=1,2,4$, 8, with the inner product $\langle x, y\rangle$ and the norm $N x=\langle x, x\rangle=\bar{x} x=x \bar{x}$ where $x \mapsto \bar{x}$ is the standard involution of $X$. We put $T x=\bar{x}+x$, the trace of $x$. Then, we have

$$
\langle x, y\rangle=1 / 2 T(\bar{x} y) .
$$

The following properties of the trace

$$
T(x y)=T(y x), \quad T((x y) z)=T(x(y z))
$$

are very useful because the algebra $X$ itself is not necessarily commutative and associative.

Let $f_{t}, t>-1$, be the quadratic map $R^{2 p}=X \times X \rightarrow R^{1+p}=R \times X$ defined by

$$
f_{t}(z)=\left(N x-N y, 2(1+t)^{1 / 2} x y\right), \quad z=(x, y) \in R^{2 p}=X \times X .
$$

1) See [1] p. 233, line 2. 
When $t=0, f_{0}$ induces a map of $S^{2 p-1}$ onto $S^{p}$ which is the classical Hopf fibration.

(3.4) Proposition. For each $t>-1$, the map $f_{t}: \boldsymbol{R}^{2 p} \rightarrow \boldsymbol{R}^{1+p}$ defined by (3.3) is of type (S).

Proof. Let $A_{t}$ be the symmetric matrix in $\boldsymbol{R}_{2 p}$ such that

$$
\left\langle\zeta, f_{t}(z)\right\rangle=A_{t}[z], \quad z=(x, y) \in X \times X, \quad \zeta=(\xi, \eta) \in R \times X .
$$

Substituting (3.3) in the left hand side, we have

$$
\left\langle\zeta, f_{t}(z)=\xi(N x-N y)+2(1+t)^{1 / 2}\langle\eta, x y\rangle .\right.
$$

By (3.1), (3.2), we have

$$
\langle\eta, x y\rangle=\frac{1}{2} T(\bar{\eta}(x y))=\frac{1}{2} T(\overline{x y} \eta)=\frac{1}{2} T(\bar{y}(\bar{x} \eta))=\frac{1}{2} T(\bar{x}(\eta \bar{y}))=\langle x, \eta \bar{y}\rangle .
$$

Since, for each $\eta \in X$, the map $y \mapsto \eta \bar{y}$ is a linear endomorphism of $X$, there is a matrix $B(\eta)$ in $\boldsymbol{R}_{p}$ such that

$$
\eta \bar{y}=B(\eta) y, \quad \text { for all } y \in X .
$$

Hence, from (3.5), (3.6), it follows that

$$
\left\langle\xi, f_{t}(z)\right\rangle=\xi(N x-N y)+2(1+t)^{1 / 2}\langle x, B(\eta) y\rangle .
$$

From this, one verifies easily that

$$
\left\langle\zeta, f_{t}(z)\right\rangle=A_{t}[z] \quad \text { with } \quad A_{t}=\left(\begin{array}{cc}
\xi_{p}^{1} & (1+t)^{1 / 2} B(\eta) \\
(1+t)^{1 / 2} B(\eta) & -\xi_{p}^{1}
\end{array}\right) .
$$

Therefore, $f_{t}$ satisfies (S1), (S2) of (2.4). Next, we shall show that

$$
{ }^{t} B(\eta) B(\eta)=B(\eta)^{t} B(\eta)=(N \eta)^{1_{p}}
$$

In fact, using (3.1), (3.2), (3.7), we see that

$$
\begin{aligned}
\left\langle{ }^{t} B(\eta) x, y\right\rangle & =\langle x, B(\eta) y\rangle=\langle x, \eta \bar{y}\rangle=\frac{1}{2} T(\bar{x}(\eta \bar{y})) \\
& =\frac{1}{2} T(\bar{y}(\bar{x} \eta))=\langle y, \bar{x} \eta\rangle=\langle\bar{x} \eta, y\rangle, \quad \text { for all } y \in X,
\end{aligned}
$$

which implies that

$$
{ }^{t} B(\eta) x=\bar{x} \eta
$$

From (3.7), (3.9), we have

$$
\begin{aligned}
& { }^{t} B(\eta) B(\eta) y={ }^{t} B(\eta) \eta \bar{y}=(y \bar{\eta}) \eta=(N \eta) y, \\
& B(\eta)^{t} B(\eta) x=B(\eta) \bar{x} \eta=\eta(\bar{\eta} x)=(N \eta) x,
\end{aligned}
$$


which proves (3.8). It follows from (3.8) that

$$
A_{t}^{2}=a 1_{2 p} \quad \text { with } \quad a=a(\zeta)=\xi^{2}+(1+t) N \eta .
$$

Since $a(\zeta)$ is positive definite for $t>-1, f_{t}$ satisfies (S3) of (2.4). Q.E.D.

Having verified that $f_{t}$ is of type (S), we may use (2.7), with $m=p$ +1 , and get

$$
N_{k}\left(f_{t}\right)=\int_{S^{p}} a^{k} d \omega_{p}
$$

Since $\xi^{2}+N \eta=N \zeta=1$ on $S^{p}$, we have

$$
N_{k}\left(f_{t}\right)=\int_{S^{p}}(1+t N \eta)^{k} d \omega_{p}=\sum_{j=0}^{k}\left(\begin{array}{l}
k \\
j
\end{array}\right) t^{j} \int_{S^{p}}(N \eta)^{j} d \omega_{p} .
$$

Now, the eigenvalues of the quadratic form $N \eta$ on $R^{p+1}$ are $\mu=(0,1, \cdots$, 1) $\in \boldsymbol{R}^{p+1}$, and so, by (1.2), (1.3),

$$
\int_{S^{p}}(N \eta)^{j} d \omega_{p}=\frac{b_{j}(2 ; \mu)}{b_{j}\left(2 ; 1_{p+1}\right)}=\frac{j !}{4^{j}((p+1) / 2, j)} b_{j}(2 ; \mu) .
$$

On the other hand, since

$$
\prod_{i=1}^{p+1}\left(1-4 \mu_{i} t\right)^{-1 / 2}=(1-4 t)^{-p / 2}=\sum_{j=0}^{\infty}\left(\frac{p}{2}, j\right) \frac{4^{j} t^{j}}{j !},
$$

by (1.1), we have

$$
b_{j}(2 ; \mu)=\frac{(p / 2, j) 4^{j}}{j !}
$$

Therefore, we have

$$
\begin{aligned}
N_{k}\left(f_{t}\right) & =\sum_{j=0}^{k}\left(\begin{array}{l}
k \\
j
\end{array}\right) t^{j} \frac{(p / 2, j)}{((p+1) / 2, j)}=\sum_{j=0}^{k} \frac{(-k, j)(p / 2, j)}{((p+1) / 2, j)} \frac{(-t)^{j}}{j !} \\
& ={ }_{2} F_{1}\left(-k, \frac{p}{2} ; \frac{p+1}{2} ;-t\right) .
\end{aligned}
$$

Finally, we have

$$
\begin{aligned}
f_{t}^{\#}(z) & =\sum_{k=0}^{\infty} N_{k}\left(f_{t}\right) \frac{z^{k}}{k !}=\sum_{k=0}^{\infty} \frac{z^{k}}{k !} \sum_{j=0}^{\infty} \frac{(-k, j)(p / 2, j)}{((p+1) / 2, j)} \frac{(-t)^{j}}{j !} \\
& =\sum_{j=0}^{\infty} \frac{(p / 2, j)(-t)^{j}}{((p+1) / 2, j) j !} \sum_{k=0}^{\infty} \frac{(-k, j)}{k !} z^{k},
\end{aligned}
$$

where the inner sum is equal to 


$$
\sum_{k \geqq j}(-1)^{j}\left(\begin{array}{l}
k \\
j
\end{array}\right) \frac{j !}{k !} z^{k}=(-1)^{j} \sum_{k \geqq j} \frac{z^{k}}{(k-j) !}=(-1)^{j} z^{j} e^{z}
$$

Therefore, we obtain

$$
f_{t}^{\sharp}(z)=e_{1}^{z} F_{1}\left(\frac{p}{2} ; \frac{p+1}{2} ; t z\right) .
$$

\section{REFERENCES}

[1] W. Magnus, F. Oberhettinger, R. P. Soni, Formulas and Theorems for the Special Functions of Mathematical Physics, 3rd Ed., Springer-Verlag, New York, 1966.

[2] T. Ono, On a generalization of Laplace integrals, Nagoya Math. J., 92 (1983), 133-144.

\section{Department of Mathematics}

The Johns Hopkins University

Baltimore, Maryland, 21218

U.S.A. 